\title{
Measurement of neuronal activity in a macaque monkey in response to animate images using near-infrared spectroscopy
}

\author{
Masumi Wakita', Masahiro Shibasaki' , Takashi Ishizuka², Joerg Schnackenberg ${ }^{2}$, Michiyuki Fujiawara² and \\ Nobuo Masataka ${ }^{*}$
}

1 Primate Research Institute, Kyoto University, Inuyama, Japan

2 Hitachi Medical Corporation, Tokyo, Japan

Edited by:

Inga D. Neumann,

University of Regensburg, Germany

Reviewed by:

Peter Eichhammer,

University of Regensburg, Germany

Michael Plichta,

University Hospital Wuerzburg,

Germany

*Correspondence:

Nobuo Masataka, Primate Research Institute, Kyoto University, Inuyama,

Aichi 484-8506, Japan.

e-mail:masataka@pri.kyoto-u.ac.jp
Near-infrared spectroscopy (NIRS) has been used extensively for functional neuroimaging over the past decade, in part because it is considered a powerful tool for investigating brain function in human infants and young children, for whom other neuroimaging techniques are not suitable. In particular, several studies have measured hemodynamic responses in the occipital region in infants upon exposure to visual stimuli. In the present study, we used a multi-channel NIRS to measure neuronal activity in a macaque monkey who was trained to watch videos showing various circus animals performing acrobatic activities without fixing the head position of the monkey. Cortical activity from the occipital region was measured first by placing a probe comprising a $3 \times 5$ array of emitters and detectors $(2 \times 4 \mathrm{~cm}$ ) on the area (area 17), and the robustness and stability of the results were confirmed across sessions. Cortical responses were then measured from the dorsofrontal region. The oxygenated hemoglobin signals increased in area 9 and decreased in area $8 \mathrm{~b}$ in response to viewing the videos. The results suggest that these regions are involved in cognitive processing of visually presented stimuli. The monkey showed positive responsiveness to the stimuli from the affective standpoint, but its attentional response to them was an inhibitory one.

Keywords: near-infrared spectroscopy, neuroimaging, macaque monkey, affective salience, animate categorization

\section{INTRODUCTION}

The visual system of primates is remarkably efficient for analyzing information about objects present in complex natural scenes. Investigations with macaque monkeys have demonstrated that these animals can efficiently categorize targets such as animals (see Grand et al., 2008 for review). In some of those studies, for instance, monkeys responded rapidly by touching a screen when a target object was present in an image. However the cognitive role of the salient features of the attractive stimuli is understood only poorly. According to previous literature about humans (Masataka, 2003), one could broadly hypothesize two possibilities regarding such salient features, that is, attention-getting properties and affective salience. The attentional responsiveness of the monkeys might be enhanced by the stimuli and/or the monkeys might respond to the stimuli positively from an affective standpoint. The present experiment was undertaken in order to investigate whether both or either of these possibilities could be confirmed by measuring the neuronal activity of a macaque monkey while it was being exposed to videos of animals performing acrobatic activities. For the measurement, we used near-infrared spectroscopy (NIRS).

Near-infrared spectroscopy can be used to monitor brain activity by identifying changes in the concentrations of oxygenated (oxy-) and deoxygenated (deoxy-) hemoglobin ( $\mathrm{Hb}$ ) and by measuring the changes in the concentrations under diffuse transmittance of NIRS light at an appropriate combination of wavelengths. Multichannel continuous wave NIRS monitors have been used extensively for functional neuroimaging over the past decade (Koizumi et al., 2003), in part because they are considered powerful tools for investigating brain function in human infants and young children, for whom other neuroimaging techniques are not suitable (Hoshi, 2003; Obrig and Villringer, 2003).

Near-infrared spectroscopy enables reliable measurements of localized hemodynamic response in infants as well as adults (Pena et al., 2003). In particular, several studies have measured hemodynamic responses in the occipital region in infants upon exposure to visual stimuli (see Minagawa-Kawai et al., 2008 for review). The primary advantage of using NIRS to assess brain activity in infants is that the technique can be performed under fewer body constraints than can other imaging modalities. In contrast, functional magnetic resonance imaging (fMRI) and electrophysiologic recording require that the subject maintain an unusual body posture, such as the sphinx position in monkeys or the supine position while performing cognitive tasks in humans, and head movement must be severely restricted. Moreover, fMRI emits a loud noise that is not only uncomfortable but may interfere with interpretation of the results. In this respect, the conditions for performing NIRS measurements are much more comfortable for human as well as nonhuman subjects. Therefore, despite the decreased temporaland spatial-resolution and depth of the recording compared to fMRI and electrophysiologic recordings, respectively, NIRS is useful for studying brain activity under more 'natural' conditions. In the present study, we used NIRS to study brain activity in the macaque monkey (Macaca mulatta) without restricting head movement. There are two previous reports of NIRS studies in macaque monkeys, in which head movement was restricted by surgical fixation and the recordings were made with single- or few-channel NIRS 
(Fuster et al., 2005; Radhakrishnan et al., 2009). Here, we used a multi-channel NIRS so that responses from a wider region of the cortical surface could be recorded simultaneously.

Cortical activity of the occipital region was recorded first because several studies in which NIRS measurements were used report event-related activation in the occipital region of human infants in response to visual stimulation (Taga et al., 2003; Csibra et al., 2004). The visual response in the occipital area was robust to any kind of stimulus. We examined test-retest reliability of the hemodynamic responses in the region because such stability has been becoming the tighter demonstration of the phenomenon in humans (Plichta et al., 2006). We then attempted to conduct the same measurement from the prefrontal cortex to evaluate frontal lobe activity in the same animal under the same experimental setting, as has been conducted in humans (Schecklmann et al., 2008).

A recent NIRS study in human adults reported activation of the prefrontal cortical region upon exposure to the visual presentation of socially relevant movie stimuli (Leon-Carrion et al., 2007). Prefrontal activity in infants viewing affective visual stimuli has also been reported (Watanabe et al.2008). The findings of these studies suggest that regional differences in prefrontal activity are likely due to both the affective salience of the stimuli and the attentional effort of the participants. Therefore, we reasoned that significant hemodynamic changes might be observed around this cortical area in the macaque upon visual presentation of stimuli if the stimuli were sufficient to hold the attention of the animal. Based on preliminary trials showing that the attention of a macaque was effectively evoked by a movie of various circus animals performing acrobatic activities, we elected to use this stimulus in the present study.

\section{MATERIALS AND METHODS SUBJECT}

One adult male rhesus monkey (M. mulatta) weighing $6 \mathrm{~kg}$ served as the subject. This monkey had participated in several visual studies and was therefore highly familiar with the experimental settings. Daily food and water consumption was not restricted for the experiment.

All animal care and experimental protocols are consistent with the Guide for the Care and Use of Laboratory Primates and were approved by the Institutional Animal Care and Use Committee of Primate Research Institute, Kyoto University. Nonhuman primates are an important experimental model in the investigation of the neural mechanism underlying visual cognition. The brain regions corresponding to the cognition are very similar to those of humans. Macaque monkeys are not endangered, and are in common use in many laboratories studying visual cognition, which allows the efficient comparison of related experiments. We have undertaken the present experiment with a monkey, having minimized the restriction of its body movement without any surgical operation. We took great care that the animal was comfortable and remained in good health. The animal was maintained under a controlled photo-period (12L:12D, lights on 0700 hours and lights off on 1900 hours) and was fed a single daily meal at 1000 hours that consisted of pellet food and supplemented with a variety of vegetables and fruits. The temperature in the animal room with provision for continuous change of fresh air ranged from 22 to $25^{\circ} \mathrm{C}$.

\section{BEHAVIORAL CONTROL}

The experiment was performed in a quiet, darkened room to minimize distraction. Each trial consisted of a 10-s stimulation period followed by a dark 20-s rest period. Ten trials were conducted during each recording session. During the stimulation period, we presented a high-contrast colorful movie of animal circuses (Le 24ème Festival International du Cirque du Monte-Carlo and Le 7ème Festival International du Cirque de Massy), in which the acrobatic performances of various animals, for example, cats, horses, tigers, and elephants, many of whom wore brilliantly colored costumes, were filmed. It was presented on a 10 -inch TV monitor placed $40 \mathrm{~cm}$ from the monkey's head. Thus, the stimulus size was $27 \times 20^{\circ}$. Stimuli were clipped from an identical video movie, and the monkey was always exposed to different clips. No special task was required of the animal. Juice was delivered at the offset of the stimulus, however, to keep the monkey sitting calmly in the primate chair. Head and eye movements were not controlled.

\section{NIRS PROCEDURE}

Changes in $\mathrm{Hb}$ concentrations were measured using an ETG-4000 optical topography system (Hitachi Medical Co., Japan) with a custom-made NIRS probe comprising seven detectors and eight emitters arranged in a $3 \times 5$ configuration. The inter-optode distance was $1 \mathrm{~cm}$. On the basis of a theoretical model about nearinfrared light propagation (Okada et al., 1997), we estimated that the cortical activity up to $5 \mathrm{~mm}$ below the scalp could be detected with this distance. Thus, cortical activity from a $2 \times 4 \mathrm{~cm}$ region was measured by a 22-channel array. The NIRS probe was fastened to the head using a flexible harness (Figure 1).

Cortical activity was measured from a wide occipital or frontal region. The main purpose of the occipital recording was to test the reproducibility and stability of the data. To record from this region, the bottom edge of the probe holder was aligned to the posterior occipital protuberance. The frontal region was recorded to evaluate the applicability of the current protocol to future imaging studies of frontal cognitive function. To record frontal activity, the

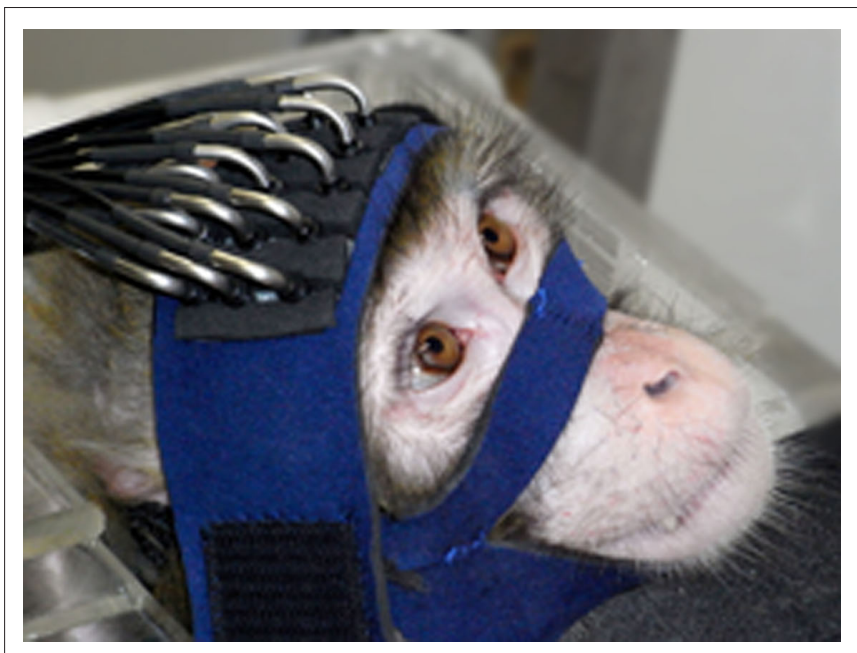

FIGURE 1 |The subject monkey with the NIRS probe. The NIRS probe was fixed to the head by a flexible band for the frontal measurement. The inter-optode distance was $1 \mathrm{~cm}$. 
anterior edge of the probe holder was set at the posterior end of the supraorbital ridge. During the measurements, the animal's face was monitored using an external video camera. The video recording was synchronized with the measurements and the footage was used to select individual tasks suitable for subsequent analysis.

The NIRS probe is conventionally secured to the head with an elastic net tubing in both macaques and human infants. To record occipital activity, however, the probe had to be attached almost vertically onto the back of the head, which could not be achieved with the net tubing because each time the monkey moved its head, the probe holder fell off the head. We therefore devised a flexible harness that swaddled the head like a muzzle. Using this harness, the position of the probe could be fairly well stabilized. Although all the optodes could not be fitted to the scalp using this method, the animal appeared comfortable and the band was well tolerated. Data from the fitted optodes were reliably integrated over multiple trials with improved signal intensity.

To determine the recording parameters, both the duration of the stimulation $(5,10$, and $20 \mathrm{~s})$ and inter-stimulus interval (ISI; $10,20,30 \mathrm{~s}$ ) were varied in the preliminary experiment. With a 5-s stimulation, the signal continued to increase even after the stimulus was terminated. Both the 10- and 20-s stimulations, however, resulted in signal changes that reached the peak level during the stimulation period. The 10 -s stimulus duration was selected for the subsequent experiments. It is crucial to keep the animal calm during the ISI. Due to a slow change in the hemodynamic response, body movement at any period during the 10-s ISI influenced the baseline calculation. A 30-s ISI was also ineffective because the monkey became restless. Therefore, a 20-s stimulus ISI was selected.

\section{NIRS DATA ANALYSIS}

Based on information from the video footage taken of the monkey during the measurements, data obtained during periods when the animal failed to watch the stimuli during the stimulation period were excluded from the analysis. Consequently, all data from six of 10 occipital recording sessions were not analyzed. In addition, of the remaining four sessions, data from third, fifth, seventh, and fourth trials, respectively, were excluded from further analysis. For the frontal recordings, data from eight trials from a single session were analyzed. The exclusion was undertaken solely due to such technical reasons. After removing unsuitable trials from each session, a baseline correction of the continuously sampled data was performed to obtain a clearer image of the entire measurement. Oscillating noise, caused by heartbeat and blood flow was removed by applying a low pass filter $(0.5 \mathrm{~Hz})$. As a final step, the mean of all suitable trials was calculated for each session and, then, the computed score was averaged across session.

For analyses of averaged brain activation, moreover, waveforms recorded from each individual channel were averaged across all of the suitable 19 trials in occipital recordings and all of the 8 trials in frontal recordings, respectively. 'Baseline activity' was designated as signal values obtained during a 5 -s period immediately prior to the stimulus onset in which 50 time points were included since the brain activity was recorded at a sampling rate of $10 \mathrm{~Hz}$. "Stimulus related activity" was designated as signal values obtained during a 10 -s stimulation period in which 100 time points were included. Then, the overall averaged value for baseline activity and the aver- aged value for stimulus related activity were statistically compared for each of the occipital and of the frontal recordings, using a paired $t$-test. On the basis of these statistical results, a $t$-map was created for the occipital recording sessions and the prefrontal recording sessions, respectively. Concerning the analyzed data for the occipital recording, in addition, test-retest stability of signal intensity among channels across four recording sessions was evaluated using a Kendall tau rank correlation coefficient analysis. Magnetic resonance images of a body-weight matched monkey and a stereotaxic atlas were used to estimate the location of the recording channels on the cerebral cortical surface.

\section{RESULTS}

Immediately prior to beginning the occipital recording sessions, we observed the monkey making jerking movements with its head as had often occurred in the preliminary experiment. Therefore, we were concerned about the stability of some the optodes on the scalp, except for those that were located around the midline of the animal's head. We continued the NIRS recordings, and such movements were no longer observed until the end of the entire experiment. The results of the occipital recordings during four individual sessions, and an averaged image and a $t$-map are shown in Figures 2, 3 and 5. An analysis of test-retest reliability revealed that the rank order of signal intensities among channels was consistent across recording sessions (total $\mathrm{Hb}, X^{2}(21)=49.30, W=.59$, $p<0.01$; oxy-Hb, $X^{2}(21)=38.44, W=0.46, p<0.05$; deoxy-Hb, $\left.X^{2}(21)=45.93, W=0.55, p<0.01\right)$. Based on the surface structure, the recording site was estimated to correspond to area 17. An increased total $\mathrm{Hb}$ concentration induced by the visual stimulation was detected only from the upper channels (channels 1, 5, 7, and 11).

The results of the frontal recordings from individual channels are presented in Figures 4 and 6. We estimated that the anterior and posterior portions of the recorded area corresponded to areas 9 and 8 b, respectively. Data obtained from the lateral channels (channels $1,5,14$, and 19 on the left, and channels $4,9,18$, and 22 on the right hemisphere) were excluded, because data from these channels would be affected by movements of the lateral muscle. In contrast to the occipital region, there was a general increase in the oxy- $\mathrm{Hb}$ signal in the anterior channels and a decrease in the oxy-Hb signal in the posterior channels. Changes in the total $\mathrm{Hb}$ concentration were almost symmetrical between hemispheres, except in a pair of anterior rows (channel 2 vs. 3 ). In summary, excitatory activation was induced in area 9 whereas activity was suppressed in area $8 \mathrm{~B}$ when the monkey was watching the movies.

\section{DISCUSSION}

Although a left-sided shift was caused by the head movement of a macaque monkey as noted above, the NIRS recordings of the cortical activity in the occipital region in response to visual stimulation in the monkey with minimal constraint were robust and stable. These results are consistent with those of the previous NIRS studies in humans, which reported that cortical activation is typically indicated as a gradual increase in the oxy-Hb signal accompanied by a symmetrical or little change in the deoxy-Hb signal (Hirth et al., 1996; Hoshi and Tamura, 1997; Obrig et al., 2002). Similar trends were also observed in the parietal and prefrontal regions 


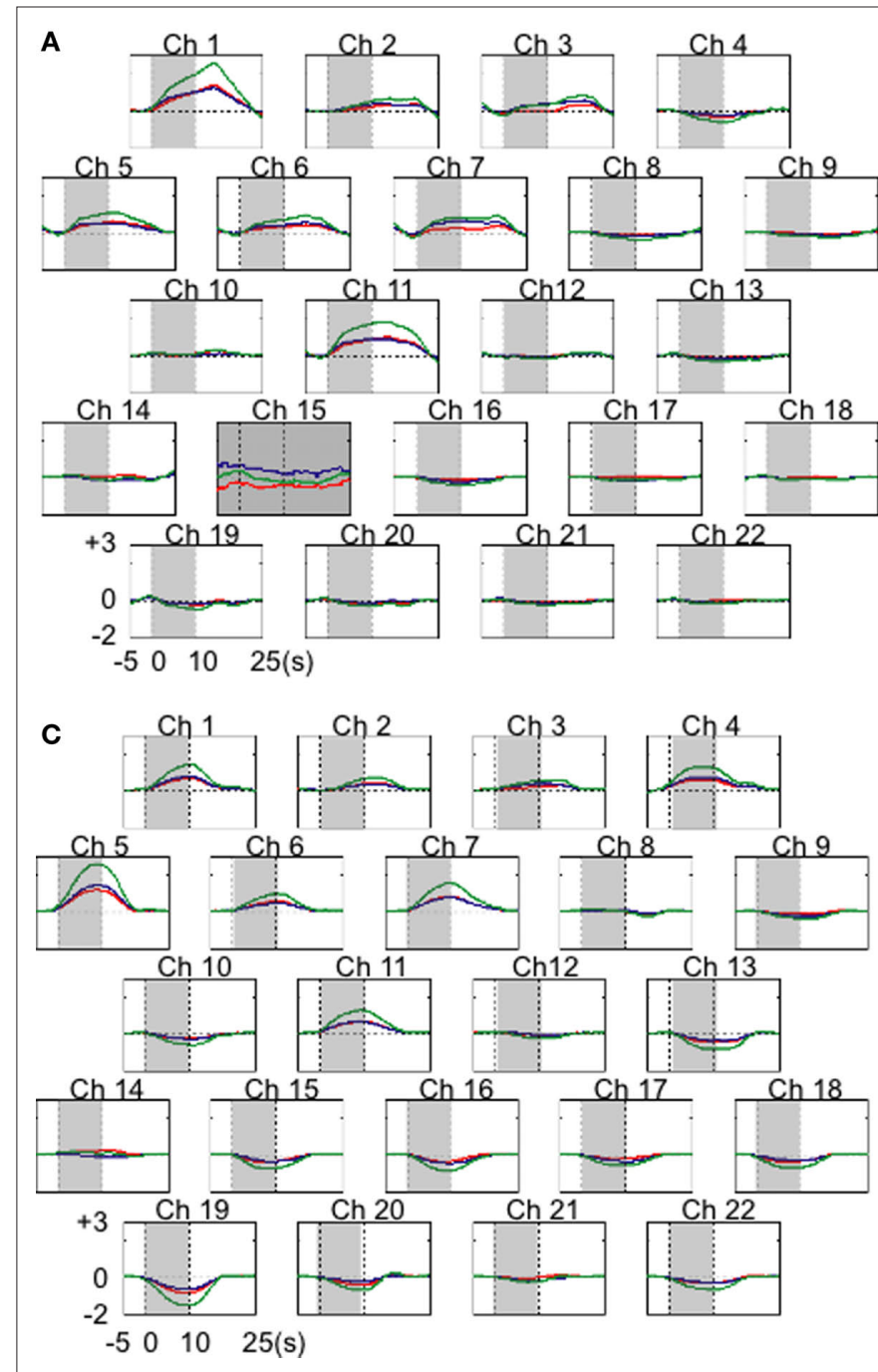

FIGURE 2 |The results of hemodynamic changes for 22 occipital cortical channels $(\mathbf{C h})$ in four individual sessions. Results from each recording session were means of third trials (A), fifth trials (B), seventh trials (C), and fourth trials (D). Channels with relatively small numbers were a located in more anterior portions of the recorded area. Changes in oxy- $\mathrm{Hb}$ are plotted in red, deoxy- $\mathrm{Hb}$ in
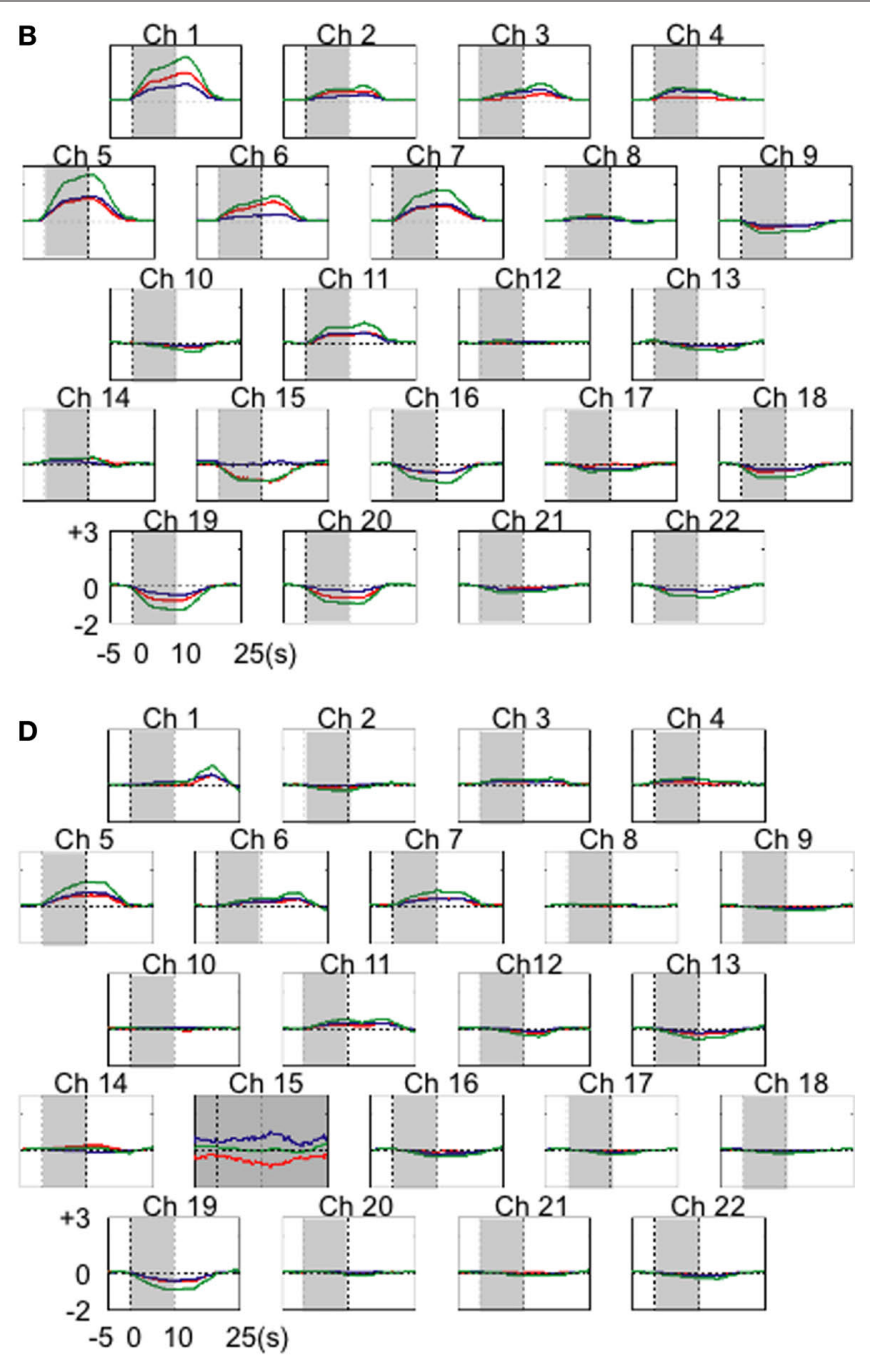

blue, and total- $\mathrm{Hb}$ in green. Shades indicate a 10-s stimulation period. Units of hemodynamic change are given as mmol-mm. Gray color of the background in Ch 15 in (A) and (D) indicates the recording of overall background noise there in the sessions. It hindered the reliable averaging of the time course of hemodynamic changes. Thus these data were excluded from further analyses. in a previous monkey study (Fuster et al., 2005). The total $\mathrm{Hb}$ concentration increased significantly only in the dorsal regions, which may be due to the fact that only optodes at the midline were appropriately fitted to the scalp and that the occipital protuberance made signals from bottom channels unsuitable. Moreover, in the present study, the hemodynamic responses in the anterior channels (area 9) were increased and those in the posterior channels (area $8 \mathrm{~b}$ ) were decreased within the frontal region during the same multi-channel NIRS measurements. Such simultaneous recording of the negative going signals as the inhibitory responses to the stimulus protocol with the positive going signals is consistent with the findings reported in a recent $\mathrm{fMRI}$ study with macaque monkeys (Vincent et al., 2007). Taken together, the present results indicate the feasibility of using NIRS to obtain hemodynamic measurements in awake macaque monkeys without surgical fixation of the head as long as the probe can be stabilized to the scalp.
Like area 9 , area $8 \mathrm{~b}$ (also called the dorsal oculomotor area) (Preuss et al., 1996), which is located anterior to the supplementary eye field, projects to the supplementary eye field (Wang et al., 2005). Nonetheless, there are reciprocal connections between area $8 \mathrm{~b}$ and parts of the occipital, parietal, and inferotemporal cortex, while area 9 does not project to any of these posterior cortical regions (Petrides and Pandya, 1984, 1999, 2006; Cavada and Goldman-Rakic, 1989; Andersen et al., 1990). Thus, area $8 \mathrm{~b}$ interacts directly with cortical regions that regulate attention to sensory events occurring in external space and, as indicated by the anatomic connectivity, shows robust activation during oculomotor tasks regardless of the presence of a visual target (Mitz and Godschalk, 1989; Schlag et al., 1992). In addition, eye and ear movements are elicited by microstimulation of this region in macaques, suggesting possible involvement of the region in the spontaneous control of such movements (Bon and 

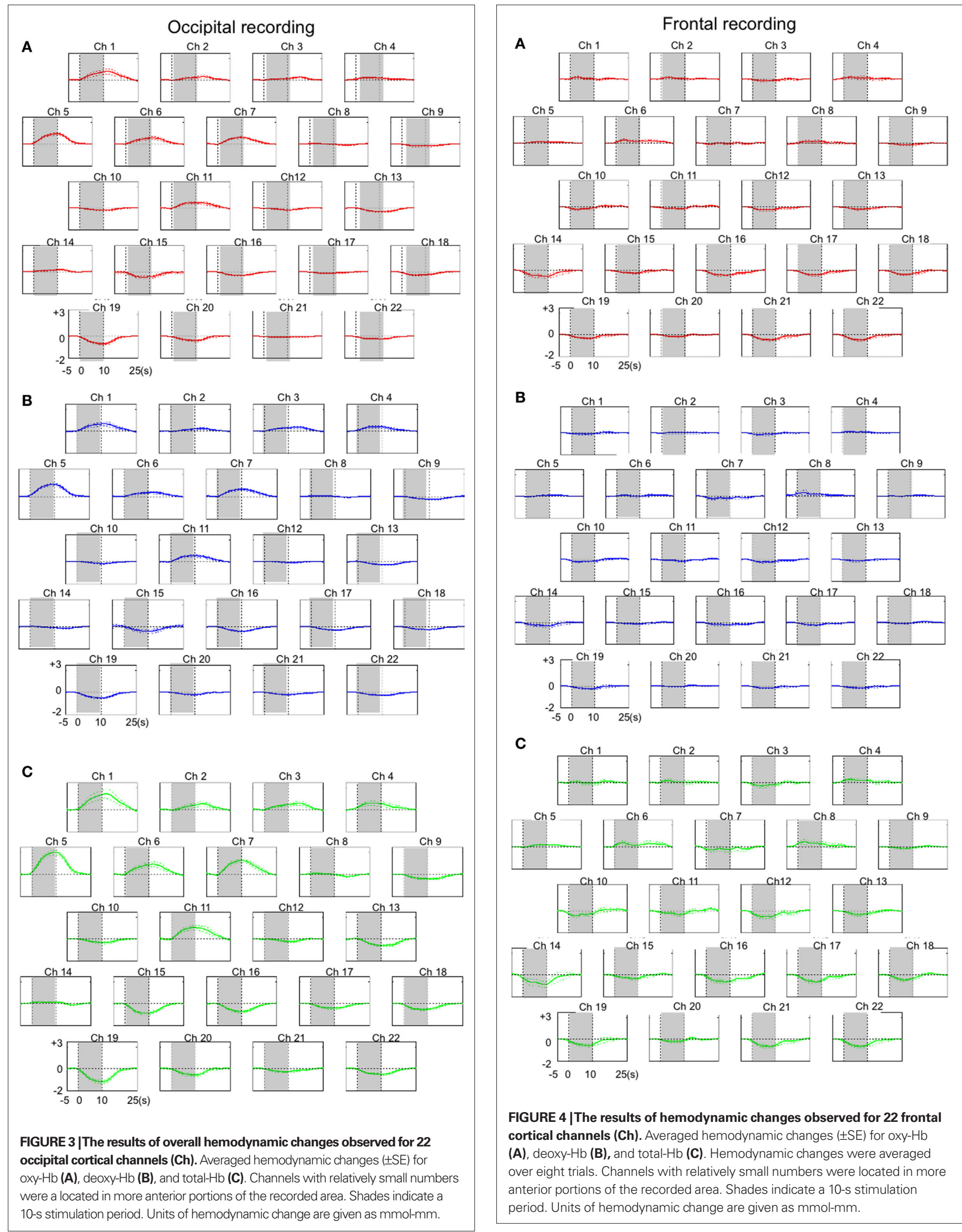

FIGURE 4 |The results of hemodynamic changes observed for 22 frontal cortical channels (Ch). Averaged hemodynamic changes $( \pm S E)$ for oxy-Hb (A), deoxy- $\mathrm{Hb}$ (B), and total- $\mathrm{Hb}$ (C). Hemodynamic changes were averaged over eight trials. Channels with relatively small numbers were located in more anterior portions of the recorded area. Shades indicate a 10-s stimulation period. Units of hemodynamic change are given as mmol-mm. 

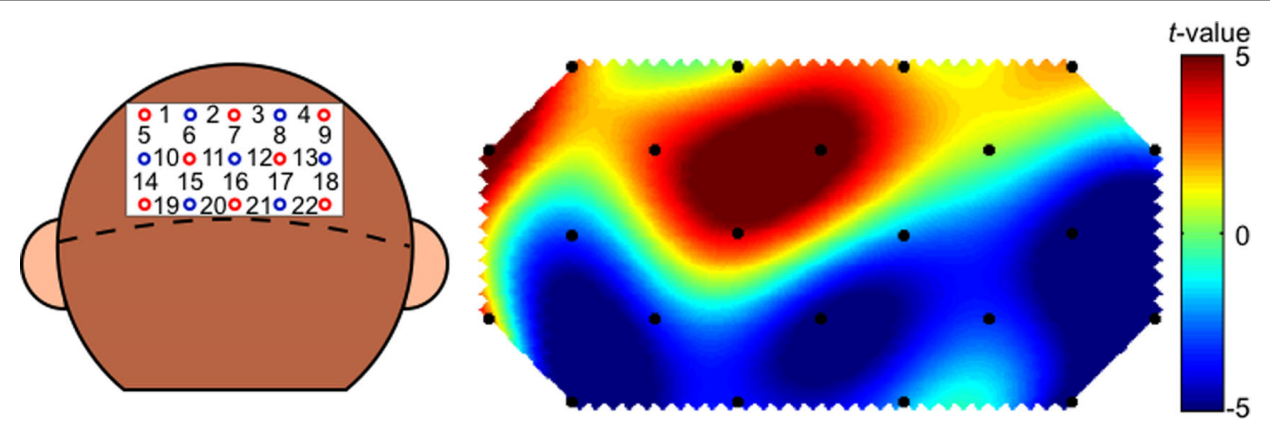

FIGURE 5 | Channel configuration over the head of the monkey for 22 occipital cortical channels (Ch) and a $t$-map created on the basis of the statistical results of comparisons of oxy- $\mathrm{Hb}$ signals prior to the stimulus presentation and the signals during the presentation.

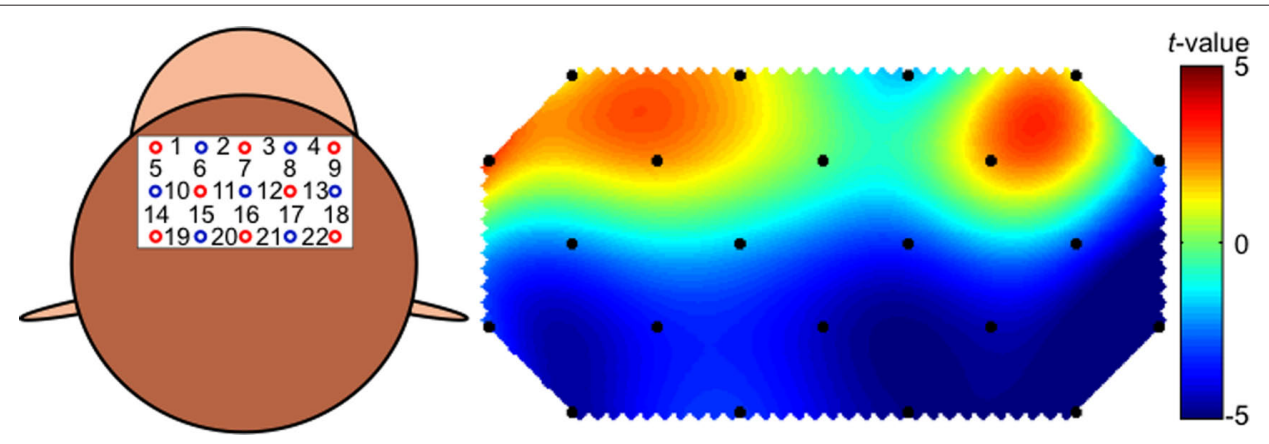

FIGURE 6 | Channel configuration over the head of the monkey for 22 frontal cortical channels (Ch) and a $t$-map created on the basis of the statistical results of comparisons of oxy-Hb signals prior to the stimulus presentation and the signals during the presentation.

Lucchetti, 1994). A suppressive response of some auditory and auditory-motor neurons has also been reported in area $8 \mathrm{~b}$ when an auditory stimulus is presented while the animal maintains fixation on a visual stimulus (Lucchetti et al. 2008). A similar inhibitory effect is observed in posterior parietal cortical activity in response to simultaneous presentation of visual and auditory stimuli (Bell et al., 2003). Together, these findings indicate that the observed decrease in the oxy- $\mathrm{Hb}$ signal in area $8 \mathrm{~b}$ may reflect attentional processes. In the present experiment, there was no visual target on which the animal was trained to fixate. Moreover, many potential targets (various animals, theater settings, humans) appeared simultaneously so that the animal was obliged to attend to them strongly but diffusively. These experimental features may have induced a suppressive response in area $8 \mathrm{~b}$, which would link to inhibited attentional response. Of course, it should be noted that the linking requires at least two prerequisites. First, NIRS like other oxygenation-based techniques is dependent upon the tight coupling between changes in cerebral electrical activity and local blood oxygenation. While this relationship has been considered to be linear, recent studies question this linearity (Obrig et al., 2002; Sheth et al., 2004). Using NIRS in conjunction with electrophysiological methods may allow to really proof linear relationship in this experimental design (Fuster et al., 2005). In addition, there remains the possibility that decreased activity in area $8 \mathrm{~b}$ may derive from decreased activity of inhibitory-acting neurons, thereby leading finally to increased attentional response. Apparently these are issues that are to be pursued in the future.
Area 9, on the other hand, is not involved in regulating attention to events occurring in external space (Petrides and Pandya, 2007). The region does, however, have a mutual projection with superior temporal auditory and multisensory processing areas (Petrides and Pandya, 1988, 2007). Moreover, area 9 interacts with structures that are involved in emotion and motivation processing, such as the caudal orbitofrontal cortex, amygdala, and cingulate cortex. Therefore, area 9 provides a neural basis for interpretation of the intention and emotional state of others in monkeys as well as in humans (Damasio et al., 2000). The observed increase in the oxy-Hb signal in area 9 is considered to reflect affective responsiveness to the stimulus video. The findings are also consistent with previous researches with fMRI (see Roger et al., 2003 for review).

Because vision is one of the most important senses in macaque monkeys as well as in apes and humans, there is extensive literature on the sophisticated visual recognition and discriminatory abilities of these animals based on their performances in cognitive tasks (Mace et al., 2005; Masataka et al., 2009). Several studies have assessed how individuals perceive their animated environment and, in particular, have identified features of the environment that are significant to the animal. Since the pioneering studies of Butler and colleagues in the 1960s in which videos were used to study macaque perception, the use of videos as stimuli is one of the most frequently used methods for this purpose (Butler, 1961; Butler and Woolpy, 1963). Captive macaques show much stronger interest in films of animated objects than in films of inanimate objects, and the exploration of the material in the animated environments may, 
in itself, be rewarding (Plimpton et al., 1981). Observations of the monkey behavior in the present study are consistent with these previous claims.

Based upon such findings, video stimulation has been successfully used to enrich the environment of monkeys in captivity (Platt and Novak, 1997). There is little evidence of habituation to such stimulation in the animals. More recent experimental studies reveal that macaques have the cognitive ability to categorize species of various animals from visual images in videos almost as precisely as humans, even though the animals are unfamiliar to the macaques (Mace et al., 2005). Taken together, the results of the present successful NIRS measurement in a nonhuman animal without fixation of the head, suggest that the dorsofrontal brain region is involved in cognitive processing of visually presented animate images. Moreover, the monkey showed obviously positive responsiveness to the stimuli from the affective standpoint, but its attentional response to them was more ambiguous.

\section{REFERENCES}

Andersen, R., Asanuma, C., Essick, G., and Siegel, R. (1990). Corticocortical connections of anatomically and physiologically defined subdivisions within the inferior parietal lobule. J. Comp. Neurol. 296, 65-113.

Bell, A., Corneil, B., Munoz, D., and Meredith, M. (2003). Engagement of visual fixation suppresses sensory responsiveness and multisensory integration in the primate superior colliculus. Eur. J. Neurosci. 18, 2867-2873.

Bon, L., and Lucchetti, C. (1994). Ear and eye representation in the frontal cortex, area $8 \mathrm{~b}$, of macaque monkey: an electrophysiological study. Exp. Brain Res. 102, 259-271.

Butler, R. (1961). The responsiveness of rhesus monkeys to motion pictures. J. Genet. Psychol. 98, 239-245.

Butler, R., and Woolpy, J. (1963). Visual attention in the rhesus monkey. $J$. Comp. Physiol. Psychol. 56, 324-328.

Cavada, C., and Goldman-Rakic, P. (1989). Posterior parietal cortex in rhesus monkey: II. Evidence for segregated corticocortical networks linking sensory and limbic areas with the frontal lobe. J. Comp. Neurol. 287, 422-445.

Csibra, G., Henty, J., Volein, A., Elwell, C., Tucker, L., Meek, J., and Johnson, M. (2004). Near infrared spectroscopy reveals neural activation during face perception in infants and adults. $J$. Pediatr. Neurol. 2, 85-89.

Damasio, A., Grabowski, T., Bechara, A., Damasio, H., Ponto, L., Parvizi, J., and Hichwa, R. (2000). Subcortical and cortical brain activity during the feeling of self-generated emotions. Nat. Neurosci. 3, 1049-1056.

Fuster, J., Guiou, T., Ardestani, A., Cannestra, A., Sheth, S., Zhou, Y.,
Toga, A., and Bodner, M. (2005). Near-infrared spectroscopy (NIRS) in cognitive neuroscience of the primate brain. NeuroImage 26, 215-220.

Grand, P., Joufrais, C., and Kirchner, C. (2008). Ultra-rapid categorisation in non-human primates. Anim. Cogn. 11, 485-493.

Koizumi, H., Yamamoto, T., Maki, A., Yamashita, Y., Sato, H., Kawaguchi, H., and Ichikawa, N. (2003). Optical topography: practical problems and new applications. Appl. Opt. 42, 3054-3062.

Hirth, C., Obrig, H., Villringer, K., Thiel, A., Bernarding, J., Mühlnickel, W., Flor, H., Dirnadl, U., and Villriner, A. (1996). Non-invasive functional mapping of the human motor cortex using near-infrared spectroscopy.NeuroReport 7, 1977-1981.

Hoshi, Y. (2003). Functional near-infrared optical imaging: utility and limitations in human brain mapping. Psychophysiology 40, 511-520.

Hoshi, Y., and Tamura, M. (1997). Nearinfrared optical detection of sequential brain activation in the prefrontal cortex during mental tasks. NeuroImage

Leon-Carrion, J., Martin-Rodriguez, J., Damas-Lopez, J., Pourrezai, K., Izzetoglu, K., Martin, J., and Dominguez-Morales, M. (2007). Does dorsolateral prefrontal cortex (DLPFC) activation return to baseline when sexual stimuli cease? The role of DLPFC in visual sexual stimulation. Neurosci. Lett. 416, 55-60.

Lucchetti, C., Lanzilotto, M., and Bon, L. (2008). Auditory-motor and cognitive aspects in area $8 \mathrm{~b}$ of macaque monkey' prefrontal cortex: a premotor ear-eye field (PEEF). Exp. Brain Res. $186,131-141$. 5, 292-297.

In addition, the findings of the present study indicate the feasibility of using non-invasive NIRS measurements to investigate neural processing in macaques and other primates, such as young chimpanzees. In this context, NIRS may contribute to validate nonhuman models of those disorders that are associated with dysfunction of the prefrontal cortex such as schizophrenia, autism spectrum disorder as well as attention deficit hyperactivity disorder on a neurological basis, comparing cerebral activity in animals with corresponding activity patterns in humans suffering from these disorders.

\section{ACKNOWLEDGMENTS}

The present study was supported by a grant-in-aid from the Ministry of Education, Science, Sports and Culture, Japanese Government (\#20243034 to Nobuo Masataka) as well as by Global COE Research Program (A06 to Kyoto University). The authors gratefully acknowledge Akemi Kato for her helpful comments when conceiving and designing this experiment, and Elizabeth Nakajima for her reading the earlier version of this manuscript and correcting its English.

Mace, M., Richard, G., Delormel, A., and Fabre-Thorpe, M. (2005). Rapid categorization of natural scenes in monkeys: target predictability and processing speed. NeuroReport 16, 349-354.

Masataka, N. (2003). The Onset of Language. Cambridge: Cambridge University Press.

Masataka, N., Koda, H., Urasopon, N., and Watanabe, K. (2009). Freeranging macaque mothers exaggerate tool-using behavior when observed by offspring. PLoS One 4, e4768, doi:10.1371/journal.pone.0004768.

Minagawa-Kawai, Y., Mori, K., Hebden, J., and Dupoux, E. (2008). Optical imaging of infants' neurocognitive development: recent advances and perspectives. Dev. Neurobiol. 68 712-728.

Mitz, A., and Godschalk, M. (1989). Eyemovement representation in the frontal lobe of rhesus monkeys. Neurosci. Lett. 106, 157-162.

Obrig, H., Israel, H., Kohl-Bareis, M., Uludag, K., Wenzel, R., Müller, B., Arnold, G., and Villringer, A. (2002). Habituation of the visually evoked potential and its vascular response: implications for neurovascular coupling in the healthy adult. NeuroImage 17, 1-18.

Obrig, H., and Villringer, A. (2003) Beyond the visible-imaging the human brain with light. J. Cereb. Blood Flow Metab. 23, 1-18.

Okada, F., Firbank, M., Schweiger, M., Arridge, S., Cope, M., and Delpy, D. (1997). Theoretical and experimental investigation of near-infrared light propagation in a model of the adult head. Appl. Opt. 36, 21-31.

Pena, M., Maki, A., Kovacic, D., DehaeneLambertz, G., Koizumi, H., Bouquet,
F., and Mehler, J. (2003). Sounds and silence: an optical topography study of language recognition at birth. Proc. Natl. Acad. Sci. USA 100, 10702-10705.

Petrides, M., and Pandya, D. (1984). Projections to the frontal cortex from the posterior parietal region in the rhesus monkey. J. Comp. Neurol. 228, 105-116.

Petrides, M., and Pandya, D. (1988). Association fiber pathways to the frontal cortex from the superior temporal region in the rhesus monkey. J. Comp. Neurol. 273, 52-66.

Petrides, M., and Pandya, D. (1999). Dorsolateral prefrontal cortex: comparative cytoarchitectonic analysis in the human and the macaque brain and corticocortical connection patterns. Eur. J. Neurosci. 11, 1011-1036.

Petrides, M., and Pandya, D. (2006). Efferent association pathways originating in the caudal prefrontal cortex in the macaque monkeys. J. Comp. Neurol. 498, 227-251.

Petrides, M., and Pandya, D. (2007). Efferent association pathways from the rostral prefrontal cortex in the macaque monkey. J. Neurosci. 27, 11573-11586.

Platt, D., and Novak, M. (1997). Videostimulation as enrichment for captive rhesus monkeys (Macaca mulatta). Appl. Anim. Behav. Sci. 52, 139-155.

Plichta, M., Herrmann, M., Baehene, C., Ehlis, A., Richiter, M., Pauli, P., and Fallgatter, A. (2006). Event-related functional near-infrared spectroscopy (NIRS):Are the measurements reliable? NeuroImage 31, 116-124.

Plimpton, E., Swartz, K., and Rosenblum, L. (1981). Responses of juvenile bonnet macaques to social stimuli 
presented through color videotapes. Dev. Psychobiol. 14, 109-115.

Preuss, T., Stepniewska, I., and Kaas, J. (1996). Movement representation in the dorsal and ventral premotor areas of owl monkeys: a microstimulation study. J. Comp. Neurol. 371, 649-676.

Radhakrishnan, H., Vanduffel, W., Deng, H., Ekstrom, L., Boas, D., and Franceschini, M. (2009). Fast optical signal not detected in awake behaving monkeys. NeuroImage 45, 410-419.

Roger, B., Tootell, H., Tsao, D., and Vanduffel, W. (2003). Neuroimaging weighs in: humans meet macaques in "primate" visual cortex. J. Neurosci.23, 3981-3989.

Schecklmann, M., Ehlis, A., Plichta, M., and Fallgatter, A. (2008). Functional near-infrared spectroscopy: a longterm reliable tool for measuring brain activity during verbal fluency. NeuroImage 43, 147-155.

Schlag, J., Schlag-Rey, M., and Pigarev, I. (1992). Supplementary eye field: influence of eye position on neural signals of fixation. Exp. Brain Res. 90, 302-306.

Sheth, S., Nomoto, M., Guiou, M., Walker, M., Pouration, N., and Toga, A. (2004). Linear and nonlinear relationships between neuronal activity, oxygen metabolism, and hemodynamic responses. Neuron 42, 347-355.

Taga, G., Asakawa, K., Maki, A., Konishi, Y., and Koizumi, H. (2003). Brain imaging in awake infants by near-infrared optical topography. Proc. Natl. Acad. Sci. USA 100, 10722-10727.

Vincent, J., Patel, G., Fox, M., Snyder, A., Baker, J., Van Essen, D., Zempel, J., Snyder, L., Corbetta, M., and
Raichle, M. (2007). Intrinsic functional architecture in the anaesthetized monkey brain. Nature 447, 83-88.

Wang, Y., Isoda, M., Matsuzaka, Y., Shima, K., and Tanji, J. (2005). Prefrontal cortical cells projecting to the supplementary eye field and presupplementary motor area in the monkey. Neurosci. Res. 53, 1-7.

Watanabe, H., Homae, F., Nakano, T., and Taga, G. (2008). Functional activation in diverse regions of the developing brain of human infants. NeuroImage 43, 346-357.

Conflict of Interest Statement: The authors declare that the research was conducted in the absence of any commercial or financial relationships that could be construed as a potential conflict of interest.
Received: 11 February 2010; paperpending published: 16 April 2010; accepted: 16 May 2010; published online: 07 June 2010.

Citation: Wakita M, Shibasaki M, Ishizuka T, Schnackenberg J, Fujiawara Mand Masataka N (2010) Measurement of neuronal activity in a macaque monkey in response to animate images using near-infrared spectroscopy. Front. Behav. Neurosci. 4:31. doi: 10.3389/ fnbeh.2010.00031

Copyright (c) 2010 Wakita, Shibasaki, Ishizuka, Schnackenberg, Fujiawara and Masataka. This is an open-access article subject to an exclusive license agreement between the authors and the Frontiers Research Foundation, which permits unrestricted use, distribution, and reproduction in any medium, provided the original authors and source are credited. 